Scientific Visualization, 2020, volume 12, number 2, pages 139 - 150, DOI: 10.26583/sv.12.2.11

\title{
Digital Nutrition: Spectral Portraits of Optimal Diet
}

\author{
Y.N. Orlov',A, A.A. Kislitsyn",A, A.O. Kambarov3,B, A.K. Baturin ${ }^{4, B}$, \\ D.B. Nikituk5,B, V.A. Tutelian ${ }^{6, B}$ \\ A Keldysh Institute of Applied Mathematics RAS \\ B Institute of Nutrition and Biotechnology RAS \\ ${ }_{1}$ ORCID: https://orcid.org/oooo-ooo1-9114-0436, ov3159f@yandex.ru \\ 2 ORCID: https://orcid.org/oooo-0003-2388-0496 \\ 3 ORCID: https://orcid.org/oooo-0002-2108-2100 \\ 4 ORCID: https://orcid.org/oooo-0001-7007-621X \\ 5 ORCID: https://orcid.org/oooo-0002-4968-4517 \\ 6 ORCID: https://orcid.org/oooo-0002-4164-8992
}

\begin{abstract}
The algorithm for calculating the values of daily menu portions of dishes in accordance with certain standards is constructed. This is consumption' standards of a given set of controlled essential substances contained in food. Such substances in this work include macronutrients -proteins, fats, carbohydrates, as well as micronutrients-vitamins A, B1, B2, C, PP, carotene and the elements - sodium, potassium, calcium, magnesium, phosphorus, iron. The features of the resulting computational problems associated with the fact that the daily consumption of macronutrients and micronutrients differs in weight by 4-5 orders of magnitude are investigated.

To analyze the quality of the computational conditionality for the problem of determining the size of a dish portion, spectral portraits of the dishes nutrient composition matrices are used. The portraits show us the accuracy of calculating the matrix spectrum depending on the accuracy of the matrix elements. Such portraits are built for typical menus without specific exceptions for a conditionally healthy person and for a vegetarian. A comparative analysis of the corresponding nutrient matrices is carried out.

An algorithm for selecting dishes and calculating the values of their portions, which is stable for macronutrients, is proposed. It is shown that when the planning horizon increases, the average daily calculated consumption of micronutrients approaches to the recommended standards of the Ministry of health. It is estimated the rate of convergence on micronutrients depending on the menu type.
\end{abstract}

Keywords: the matrix of nutrients, the optimal menu, spectral portrait, the convergence of the micronutrients.

\section{Introduction}

The creation of software products for the digital nutrition involves the solution of both theoretical and practical computational problems.

The first task is to digitally transform data on human physiological needs in food and biologically active substances and energy, the chemical composition of basic food and the creation a computer program to process this data in order to create personalized recommendations for optimal nutrition [1].

The second task is in developing the combined deterministic and statistical models according to the peculiarities of nutritional characteristics, taking into account the medical recommendations, the forecast of quantitative and age-related changes in the pop- 
ulation structure, the environmental situation, and national characteristics. This is also important for predicting the regional food needs.

The third task is less global, but the accuracy of forecasts and recommendations depends on its solution, and this will be discussed in this paper. This task consists in creating an algorithm for automatic selection of dishes and calculating the size of their portions under given linear constraints (equalities and inequalities), based on some not completely formalized principle of optimality, which takes it out of the classical field of linear programming. The point is that there is no formal functionality in this task that needs to be minimized.

Optimization in this context will be understood as minimizing the amount of time required to achieve a given nutrient intake in the average daily equivalent. For example, the average annual protein intake recommended in [2] is about $100 \mathrm{~g}$ per day for an adult, of which $50 \%$ is of animal origin, and $50 \%$ is of vegetable origin (cereals, vegetables, fruits). At the same time consumption of fish and poultry meat is about $20 \%$ the total consumption of meat food, and $60 \%$ is accounted for by animal meat. Then the average daily protein intake from meat products is $10 \mathrm{~g}$ of poultry meat, $10 \mathrm{~g}$ of fish meat and $30 \mathrm{~g}$ of beef (or lamb, pork, etc.). However, it is not very natural to eat every day products that make up the average daily set. It would also be strange to eat only fish for the first quarter of the year, the second quarter to eat poultry and then animal meat. We will understand the optimality as a variety of menus, in which the average daily intake of nutrients is achieved with a given accuracy in a minimum time. This requires the development of a specific algorithm for selecting foods and dishes in accordance with their chemical composition [3] and frequency of nutrition.

Since this problem does not have an unambiguous solution, it is necessary to formalize the algorithm for selecting dishes from the existing database $[4,5]$ and calculating the portion size depending on the user's parameters.

The current state of the problem being solved is contained, in particular, in the review [6]. According to [6], there are quite similar programs, such as "Assessment of actual nutrition", "Individual diet", "Dietitian", "Nutrilogic", "Idealnutrient" and a number of others, the initial data for which are the data obtained from the patient's survey: age and anthropometric data, physical activity data, information about eating habits. For each product in the program enters the portion size and frequency of consumption. As a result, we get information about the average daily consumption. The final protocol of the program contains the calculated level of the value of the main exchange, recommendations for caloric content, the structure of indicators of food value by food groups, as well as a graph of the characteristics of food value in relation to the norms. The standards usually comply with the recommendations of the Ministry of health [2]. However, there is no description of the rules for selecting certain dishes in the daily menu in these programs.

It should also be noted that existing software products do not assume the possibility of automatically creating a menu for an arbitrary time period. At the same time, for users with a specific schedule of daily withdrawals (various types of fastings and traditional holidays), such a schedule is a practical necessity.

Note that there are accounting programs for preparing menus and accompanying accounting documents for public catering establishments that automate the functions of a dietitian, accountant-calculator or storekeeper for calculating food and accounting for the preparation of meals in the food units of medical and preventive institutions of stationary type. Such programs cannot be used for research purposes, since they do not involve menu variations in relation to the user's request. 
The main computational component of software algorithms is filling the daily menu in conditions of restrictions on the total content of nutrients. The other algorithms are related to working with databases. The optimization problem does not arise in this context.

Instead of solving the ambiguous task of creating a menu, the available programs contain the options for standard daily diets and differ mainly in the volume of databases on products and nutrients.

There are three major blocks that make up any software implementation of a particular model in the field of nutrition.

The first block is the set of models for the assimilation of food by human body (or animal, if the task relates to animal husbandry, etc.), which has certain characteristics. The basic characteristics include gender, age, height, body weight, fat mass and physical activity. Additional parameters that affect the choice of food are diseases that (if the user has them) introduce restrictions or, conversely, certain menu items, as well as the region of residence, cultural traditions, and individual preferences for certain products.

The second block is a database on the chemical composition of food. This block includes both the actual nutrient composition of the products, and the calculation of the corresponding composition, which is obtained for a dish consisting of a given set of ingredients after its preparation in a certain way. It should be emphasized that there are no reliable estimates of the accuracy of the data used. They are only available for specifically performed experiments, many of which relate to the irrelevant historical past. It is not clear how well the data used meets modern realities, even in terms of average values and variance. As a result, a priori requirements on the accuracy of incoming data for performing computational procedures, they are practically important. They show the upper limit of the error resulting from calculations of portion sizes and menu composition. An effective tool to find accuracy limits in computational linear algebra problems is the matrix's spectral portrait of the corresponding linear operator, the which construction of which is used in this work.

The third block is a combined software package of computational algorithms necessary to solve the following problem: select from the second block a set of products and dishes and determine the amount of their portions necessary to meet the vector of daily nutrient needs, which are determined in the first block by the specified anthropometric and other characteristics of the user. This section also includes a set of the statistical functions' set that allow you to perform a comparative analysis of the various menu options, determine the accuracy of solving the specified task, classify users by preferred menu types and solve other statistical problems related to nutrition science. One of the aspects of the computational program developed by the authors, related to evaluating of the accuracy of forming a rational nutrition menu, is presented in this article.

Thus, the calculated menu should be, on the one hand, quite diverse, i.e. in some sense random, and on the other - contain the necessary daily calories in the form of macronutrients. In this paper, an approach to solving such a problem is formulated and it describes some computational difficulties encountered along the way.

\section{The formulation of the problem}

Let $K$ be a total daily consumption of food and dishes. Usually their number varies from 10 to 20. Let's introduce the following notation. The vector of nutrient needs $i$ is 
considered a given value and is denoted by $F_{i}$. Both the composition of the products and the ingredients of the dishes are known. Then it is known the nutrition matrix $A_{i j}$ of mass component of nutrient $i$ in the dish $j$ at the rate of $100 \mathrm{~g}$ of the dish. Let us designate $x_{j}$ the value of $j$ dish portion. Then the equation to be solved for determining the coefficients $x_{j}$ has the form of a linear system $A \mathbf{x}=\mathbf{F}$ or

$$
\sum_{j=1}^{K} A_{i j} x_{j}=F_{i},
$$

which must be solved under condition $x_{j}>0$. The matrix $A_{i j}$ presents the main characteristics of the digital nutrition data base. Its basic minor determines the accuracy which the weight composition of the menu items is determined.

Formally, the solution of equation (1) is carried out by means of the computational linear algebra apparatus, depending on the structure of the matrix $A_{i j}$. However, in practice there may be difficulties related to poor conditioning of the food matrix.

The purpose of this paper is to describe a method that allows you to get a solution in a mathematically correct form and with reasonable coefficients. Depending on the structure of the food matrix the following solutions to the equation (1) are possible.

Variant 1. The number of menu items is exactly equal to the number of balanced nutrients $N$. The matrix $A_{i j}$ in this case is square $N \times N$. If this matrix is nondegenerate, then there is an inverse matrix $A^{-1}$, that is also considered to be known, after which the formal solution of equation (1) has the form

$$
\mathbf{x}=A^{-1} \mathbf{F} \text {. }
$$

The essence of the problem in this case is to select a menu for which the components of the found solution (the proportion of dishes in the menu) are strictly positive and are within some natural dimensions of the dish, a priori known.

Variant 2. The number menu items is less than the number of balanced nutrients. Then the matrix $A_{i j}$ has dimension $N \times K$ and the basis minor has a rang $K<N$. In general the system is incompatible in other words, it is impossible to get a solution for all the nutrients. Necessary condition for the existence of a solution is that $\operatorname{rang} A=$ rang $[A, \mathbf{F}]$, where $[A, \mathbf{F}]$ is an extended matrix, obtained from matrix $A$ by adding the vector $\mathbf{F}$ as an additional column.

If this condition, which is known from the standard course of linear algebra, is not met, you can proceed in the following way. Since the immediate goal of a daily diet is to balance calories, not microelements, the three components (protein, fat, and carbohydrate) can be determined if the number of meals is at least three. In this case, the remaining components should be determined by a wider range of consumption times - a week, two weeks, or a month. Therefore, instead of the original problem, you can solve an equation with respect to the values of portions, for example, for a menu for a week that contains at least $\mathrm{N}$ dishes, and then divide these dishes by day, taking into account the daily calorie limit. Thus, the case of the General situation in this way of solving the problem is the following variant 3 .

Variant 3. The number of $K$ menu items is greater than the number of balanced nutrients. Then the matrix $A_{i j}$ has rectangular dimensions $N \times K$, the basis minor has a rang $N$, and a system (1) in general has more than one solution. If we present the matrix $A$ in block form and designate basis minor $G$ and remain sub-matrix $U$ : 


$$
A=\left\|G_{N \times N} \quad U_{N \times K-N}\right\| .
$$

Then the equation (1) has the form:

$$
A \mathbf{x} \equiv G\left(\begin{array}{l}
x_{1} \\
\ldots \\
x_{N}
\end{array}\right)+U\left(\begin{array}{l}
x_{N+1} \\
\ldots \\
x_{K}
\end{array}\right)=\left(\begin{array}{l}
F_{1} \\
\ldots \\
F_{N}
\end{array}\right) .
$$

Thus, the coefficients of using the first $N$ dishes of the menu depend on randomly selected non-negative coefficients of the remaining $K-N$ dishes, provided that the solution is non-negative with respect to all components of $\mathbf{x}$.

Therefore, the algorithm of actions for making a daily menu consists in bringing the number of nutrients and the number of dishes to an equality. It appears as follows. Let the daily menu has $K$ dishes and let $n$ be the nearest integer to the ratio of the number of nutrients to the number of dishes $[N / K]=n \geq 1$. If $N / K>n$, then some dishes from the n-day menu are crossed out so that the condition is met $N / K=n$. If $N / K<n$, then some dishes are added to the menu of the last day. Crossing out and adding is a formal process and is not a change in the menu, but a formal change in the recipe of some dishes.

These actions lead to the formation of a menu for $n$ days, based on the calculation that the total number of dishes is equal to the number of balanced nutrients, so $N$.

A matrix is created for each such menu and calculated the inverse matrix $A^{-1}$. These objects are the database's characteristics for the chemical composition of products and dishes, and are not consumer properties, so they can be calculated in advance. After that, the problem is solved by the formula (2). However, such standard actions in practical calculations are often impossible. The reasons for this are discussed below.

\section{The nutrition matrix analysis}

Let's consider the problem of creating a menu for a healthy person with certain anthropometric parameters that correspond to a given daily intake of nutrients and, in particular, calories. In Fig. 1 we present an example of the accumulated daily caloric content for macronutrients.

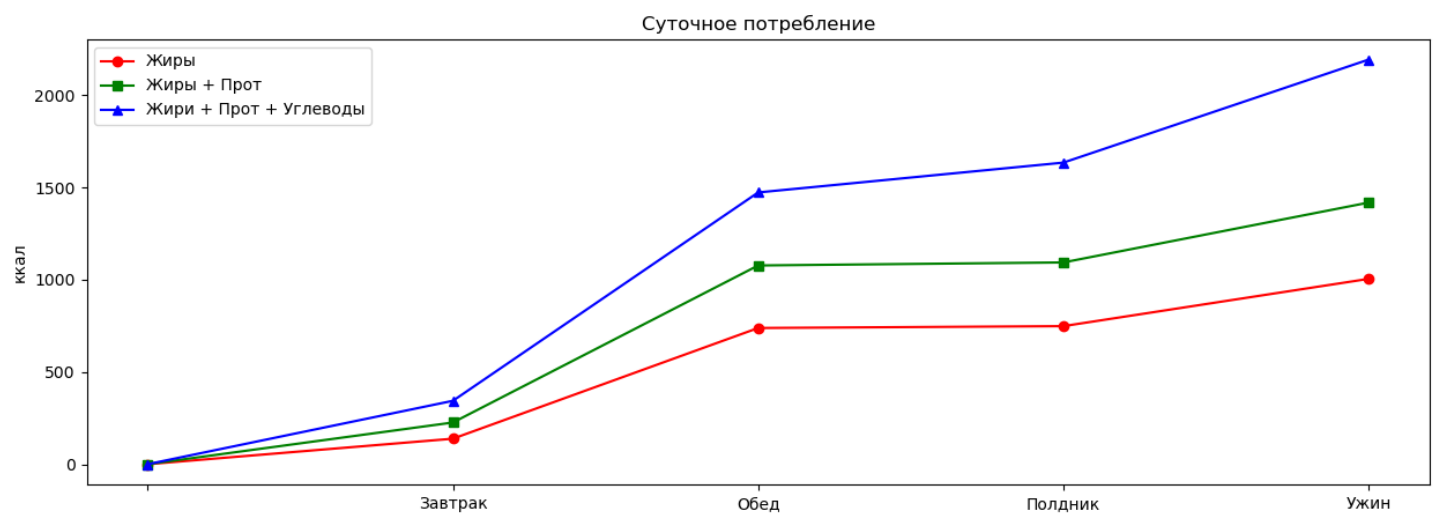

Fig. 1. Typical daily intake of essential nutrients: fat, protein and carbohydrates.

When modeling the needs vector, the following list of nutrients is used. 
Macronutrients: 1-fat, 2-protein, 3-carbohydrates.

Micronutrients: 4-vitamin C, 5-vitamin B2, 6-vitamin PP, 7-K (potassium), 8 - Ca (calcium), $9-\mathrm{P}$ (phosphorus), $10-\mathrm{Na}$ (sodium).

Micronutrients of small concentrations: 11-vitamin B1, 12-vitamin A, 13-carotene, 14$\mathrm{Mg}$ (magnesium), 15 - Fe (iron).

Let's create a menu in which the number of dishes is exactly equal to the number of balanced nutrients, without discussing the method of selecting dishes from the database. An example of this menu is shown below. Breakfast: 1-semolina porridge with milk, 2-cheese sandwich (sliced loaf, butter), 3-apricot jam, 4-black tea without sugar. Lunch: 5-Moscow borscht with meat and sour cream, 6-grain bread, 7-salad of fresh cucumbers and tomatoes with green onions and mayonnaise, 8-Lula-beef kebab with rice, 9-dried fruit compote with sugar. Afternoon tea: 10-cappuccino coffee without sugar, 11 - pastry. Dinner: 12-vegetable stew, 13-pistachios, 14-honey, 15-black tea without sugar.

However, after performing formal calculations using the formula (2), we get a vector with a negative part of its components as a solution for the portion size of such a menu, which does not make sense. The reason for this strange behavior of the solution for a seemingly acceptable menu is that the nutrition matrix is poorly defined, while the elements of the database on the chemical composition of dishes are known with relatively low accuracy (about 10-15\%), which significantly deviates from the accuracy requirements for the matrix elements for dividing spectrum points.

The computational meaning of dividing the spectrum points is the following. Assume that all eigenvalues $\lambda_{1}, \ldots, \lambda_{n}$ of the matrix $A$ are different. Corresponding eigenvectors $e_{k}$ with the conditions $A e_{k}=\lambda_{k} e_{k}, k=1, \ldots n$ form a linearly independent system of vectors that can be decomposed both vector $\mathbf{F}$ from (1) and the desired solution vector $\mathbf{x}$. Suppose that the coefficients $f_{k}$ of the expansion of the right-hand side vector are found, so that $\mathbf{F}=\sum_{k=1}^{n} f_{k} e_{k}$. Analogously $\mathbf{x}=\sum_{k=1}^{n} \alpha_{k} e_{k}$. Substituting the expansions for $\mathbf{F}$ and $\mathbf{x}$ in equation (1), we obtain a system for determining of the values $\alpha_{k}$. The solution has the form $\alpha_{k}=\frac{f_{k}}{\lambda_{k}}$. However, if the uncertainty in the elements of the matrix is such that, when calculated with this accuracy, the points of the spectrum are indistinguishable, then the eigenvalues are considered multiples, and a different formula is required to construct the solution. Spectral portrait is an effective method for determining the sufficient accuracy of input data on the studied matrix's structure to apply the above formulas.

To determine what accuracy requirements should be applied to the elements of the food matrix, we will build a spectral portrait of it. The construction of spectral portraits of matrices is based on the procedure described in the book [6]. The portrait shows the areas where the eigenvalues of matrices lie, if the elements of these matrices are known with the precision that the decimal exponent of which is specified in the legend.

In terms of resolvent of nutrition matrix $R(\lambda)=(\lambda I-A)^{-1}$ the corresponding $\varepsilon$ spectrum is defined as follows: the value $\lambda$ is belonged to $\varepsilon$-spectrum $\Lambda_{\varepsilon}(A)$, if 


$$
\|R(\lambda)\| \geq \frac{1}{\varepsilon\|A\|} .
$$

In the studying the location of the spectrum points it is convenient to consider a closed smooth curves $\gamma_{\varepsilon}$, representing the contours of $\varepsilon$-spectrum. The quality of the dichotomy $\kappa_{\gamma}(A)$ is estimated by the norm of the square of the resolvent (4) on this curve:

$$
\kappa_{\gamma}(A)=\frac{\|A\|^{2}}{l_{\gamma}} \oint_{\gamma}\|R(\lambda)\|^{2} d \lambda .
$$

Here $l_{\gamma}$ is a contour length $\gamma$. The value of $\kappa_{\gamma}(A)$ can be considered an indicator of the accuracy of spectrum separation. If there are no spectrum points $\lambda(A)$ on a certain curve $\gamma$, then the norm of the resolvent on such a curve is finite $\|R(\lambda)\|_{\gamma}<\infty$, as is the integral of it on this curve. This representation is used for numerically finding the $\varepsilon$ spectrum of the matrix. The algorithm is arranged as follows. The complex plane is divided into concentric circles in increments $\varepsilon$. For each circle the integral (5) is calculated numerically. The logarithmic growth of this integral during the transition from one circle to another indicates an approximation to the eigenvalue of the matrix $A$. The subsequent decrease in the integral (5) allows us to determine the ring, in which the eigenvalue of the matrix lies, estimated with a given accuracy $\varepsilon$. Comparing the values of the integral (5) obtained when dividing the complex plane into curves, the color of the corresponding points of this plane, we get a spectral portrait of the matrix.

The food matrices' typical spectral portraits of the traditional food' daily menu and vegan menu are shown in Fig. 2-3. From this it follows that in order to correctly solve the food equation (1), the elements of the matrix $A$ must be known at least with accuracy $10^{-3}$ (when the eigenvalues of the matrix are separated), which is not actually realized.

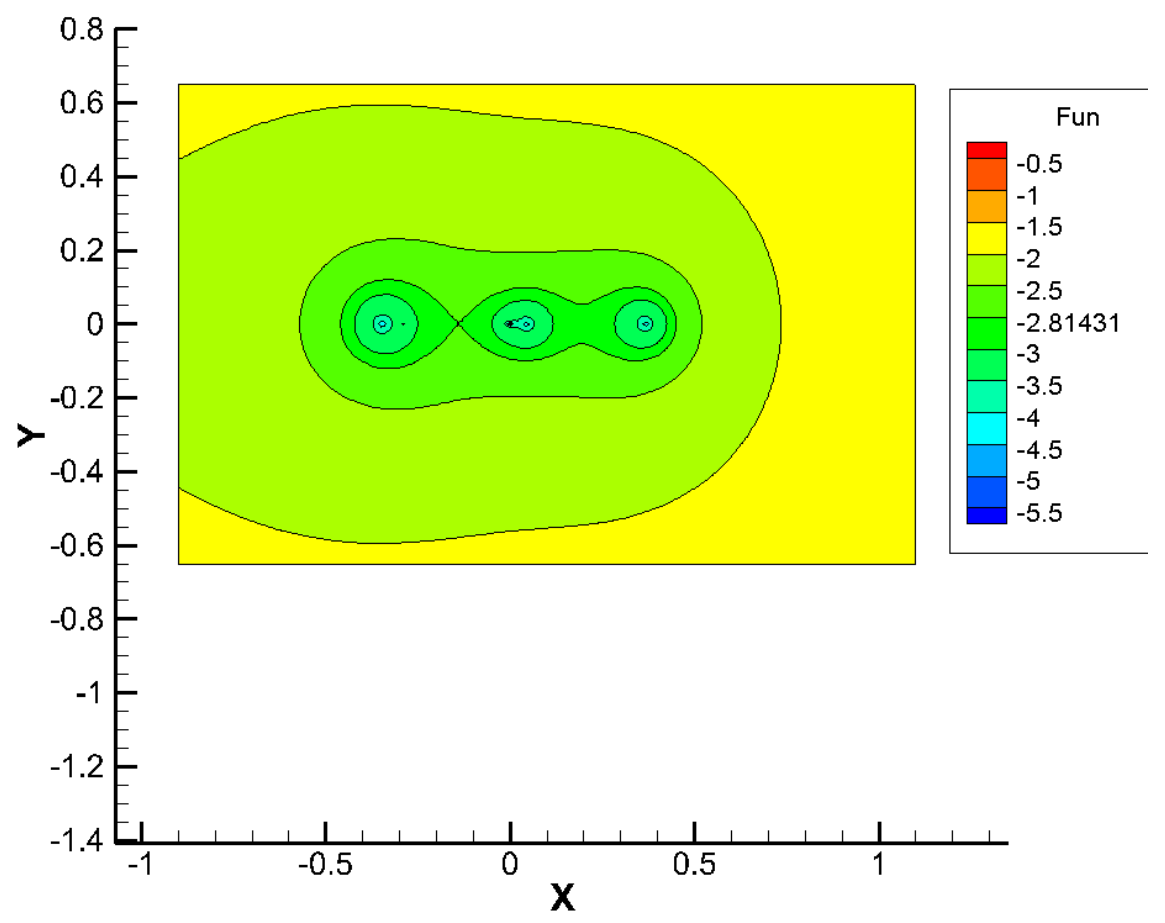

Fig. 2. Spectral portrait of traditional nutrition matrix. 


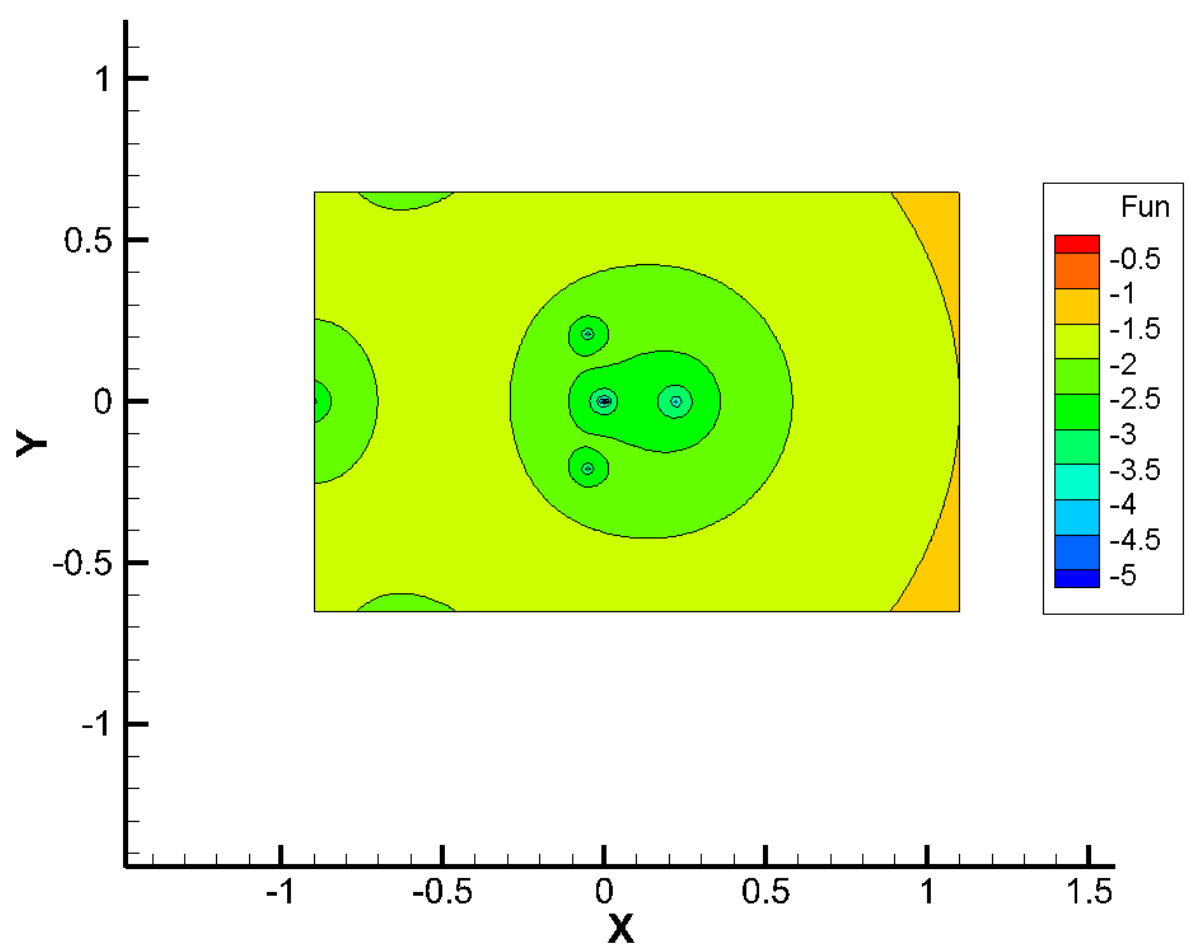

Fig. 3. Spectral portrait of vegan nutrition matrix.

The meaning of these drawings is following. If the relative accuracy of the matrix elements is $10^{-2}$ (yellow background), then the matrix has one eigenvalue (or rather, 15 matching ones) that are located somewhere in the marked square. With the accuracy $10^{-3,5}$ in Fig. 2 one can see three bluish circles containing all 15 eigenvalues, which are perceived as three in this case. In Fig. 3 the division of the spectrum points (already into four bluish circles) occurs with accuracy $10^{-3}$, i.e. the nutrition matrix of a vegan is somewhat better conditioned than that of a meat eater. However, this matrix is not applicable for calculating menu portions, since, as mentioned above, the accuracy of the matrix data of the chemical composition of dishes is no better than $10^{-1}$.

From this consideration, it also follows that increasing the number of days when calculating the menu will not change the proportion between nutrients, and therefore simultaneous calculation of the values of portions of dishes under conditions of control of all 15 nutrients at a given level of accuracy is not possible. Indirectly, this state of Affairs is evidenced by the absence of a description of the calculation algorithm in existing software products, i.e. the menu in these programs is actually built not by calculation, but by selection. The accuracy of the selection is also not specified, as is the characteristic deviation of the percentage of nutrients in certain products from the standard prescribed in the database.

\section{The solution of the problem for macronutrients}

From a computational point of view, the problem is that the menu contains dishes with a high content of macronutrients, as a result, the proportion of micronutrients in their background can't be determined with the necessary accuracy. Let's proceed as follows. Let's select the rows of the system (1) with the dominant elements related to macronutrients, and combine the menu items so that instead of a poorly conditioned 
matrix $15^{*} 15$, we get a "good" matrix $3^{*} 3$. Note that the idea of balance only for macronutrients is used in some calculations (see, for example, [8]), but without explaining the reasons for this choice.

Grouping dishes allows you to analyze the food matrix only in part of macronutrients. The spectral portrait of such a matrix is shown in Fig. 4.

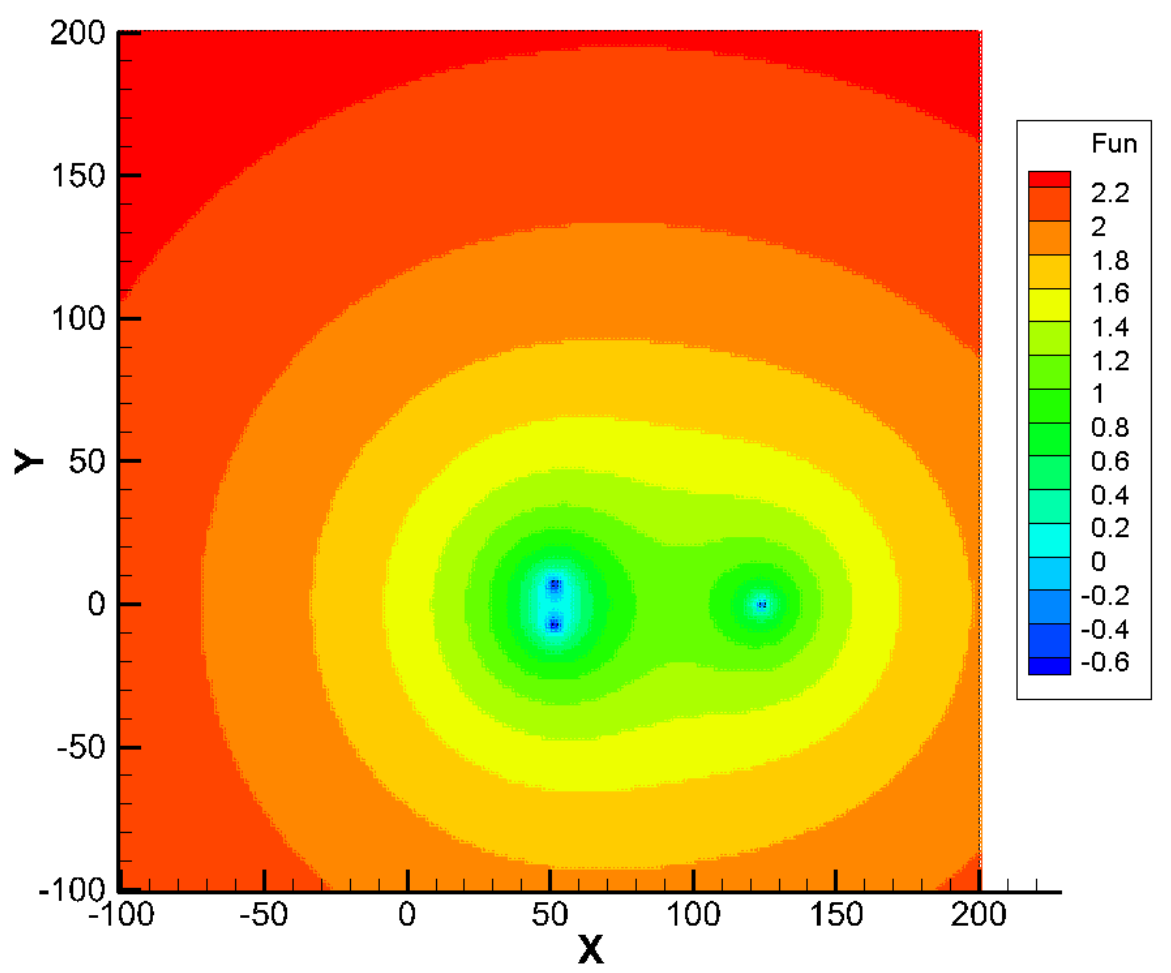

Fig. 4. Spectral portrait of the enlarged nutrition matrix.

Coordinates $X$ and $Y$ in the Fig 4 correspond to the points of the complex plane where the spectrum of the matrix $A$ can be located. Red, orange, yellow and light green areas correspond to a consistently decreasing inaccuracy (from 10 000\% to 400\%) in the matrix elements, in which its three eigenvalues are still not recognized. At an inaccuracy level of $160 \%$, the spectrum splits into two blue regions. Three eigenvalues of this matrix are identified, starting with an error of approximately 0,4 ( $\left.10^{-0,4} \approx 0,4\right)$. This means that even for very inaccurate measurements of the dishes' chemical composition, the solution of the problem for macronutrients will be correct. Let's consider the selection of dishes. A special feature of our algorithm is to create a menu with a planning horizon significantly longer than one day. It is convenient to consider a month as a planning unit. Then, based on the actual data of Rosstat the annual food consumption, you can determine their average monthly consumption. For a certain type of user, taking into account their age, gender, anthropometric and other data, these average values are corrected based on statistical models that are not discussed here. After that, the number of food types consumed during the month (meat dishes, poultry dishes, etc.) is determined, each of which is the main supplier of one of the macronutrients. Since the daily menu of the dishes' types is known, it remains to choose representatives of these types. To do this, a random set of numbers is generated - the dishes' IDs of this type in the database. Then, after combining them into 
groups, the equation (1) for the enlarged food matrix is solved. As a result, macronutrients are precisely balanced, but micronutrients may have noticeable deviations from the set standards. For each nutrient we will enter a daily deviation of the calculated consumption from the set one:

$$
\Delta_{i}^{n}=\sum_{j=1}^{K} A_{i j}^{n} x_{j}^{n}-F_{i} .
$$

We also introduce the accumulated average modulus of relative deviation

$$
r_{i}^{n}=\frac{1}{n F_{i}} \sum_{k=1}^{n}\left|\Delta_{i}^{k}\right| \text {. }
$$

Menu optimization is a search of options within the types of dishes in the database, in which the value $r_{i}^{n}$ reaches the specified level of accuracy in the minimum number of days $n$. This ensures both the preservation of a given level of nutrient consumption, and a sufficient variety of nutrition. If the database on the chemical composition of dishes is sufficiently diverse and contains about a thousand different dishes, then each of the remaining micronutrients can be balanced with a relative accuracy of 0,10 in less than two weeks.

Note that in conditions of special restrictions (exceptions), the balancing of micronutrients occurs somewhat later than for food without restrictions, although the food matrix is better conditioned for menus with exceptions. We emphasize that vitamins in tablets are not considered as possible products.

\section{Conclusion}

In this paper, an algorithm for selecting database components and calculating the corresponding weight coefficients of these components under conditions of poor conditionality of the linear relationship matrix is constructed. In relation to the challenges of the digital nutrition this algorithm is applied to build daily food menu for freely definable planning horizon. The calculation visualization method used in this work is based on the construction of a spectral portrait of the nutrient matrix, has led to the need to segment the calculation in such a way as to reduce the computational error. It is shown that the optimization problem in terms of nutrition diversity can be solved by the proposed method.

\section{References}

1. David A. Bender An introduction to nutrition and metabolism // CRC Press, 2014

2. Methodical recommendations of MP 2.3.1.2432-08 "Norms of physiological requirements for energy and nutrients for various groups of the population of the Russian Federation" (approved by the chief state sanitary doctor of the Russian Federation on December 18, 2008).

3. Tutelian V.A., Samonov M.A., Kaganov B.S., Baturin A.K., Sharafetdinov H.H., Plotnikova O.A., Pavluchkova M.S. Cardboard dishes of dietary (therapeutic and prophylactic) nutrition optimized composition. - M.: National Association of Clinical Nutrition, 2008. - 448 p.

4. Tutelian V.A. The chemical composition and calorie content of Russian food. Directory. - M.: DeLi print, 2012 .-- 284 p.

5. Raper N., Perloff B., Ingwersen L., Steinfeldt L., Anand J. An overview of USDA's Dietary Intake Data System // J Food Compos Anal, 2004.17 (3-4). 
6. Portnov N.M. Electronic Doctor // Practical Dietetics, 2012, 2 (6).

7. Godunov C.K. Modern aspects of linear algebra. - Novosibirsk: Scientific Book, 1997. - 388p.

8. Grashchenkov D.V. Mathematical modeling of formulations for the organization of baby food // Food Industry, 2018, 3 (4). 